\title{
The Navigation Risk Assessment Using Wavelet Neural Network
}

\author{
Xin $\mathrm{Cao}^{\mathrm{a}}$, Shidong $\mathrm{Fan}^{\mathrm{b}}$ \\ a Nanjing Maritime Safety Administration of the People's Republic of China, Nanjing, China \\ $b$ School of Energy and Power Engineering, Wuhan University of Technology, Wuhan, China
}

\begin{abstract}
Factors that influence the security of navigation have the characteristics of dynamics, randomness, uncertainty and mutual influence, so the system of navigation security is a typical non-linear system. Due to the limitations of traditional mathematical methods solving the non-linear system, this article combines artificial neural network model and wavelet analysis to construct the wavelet neural network model for the navigation risk assessment, and puts forward the index system, weights calculation and algorithm with examples. Results shows that the wavelet neural network model for evaluating can be solved the defects such as subjective arbitrariness and the fuzzy of conclusions existing in traditional evaluation methods, and it can do better than the common artificial neural networks in fitting accuracy and convergence rate, so the application of wavelet neural network model has an important value.
\end{abstract}

Index Terms: Navigation security; risk assessment; wavelet neural network

(C) 2011 Published by MECS Publisher. Selection and/or peer review under responsibility of the International Conference on E-Business System and Education Technology

\section{Introduction}

With the economic globalization and the gradually forming of international market, sea transportation as an economic tie connecting nations plays a great role in promoting the process of global economic integration. Thus, the development of maritime industry, the expanding of ports' scale, the construction of cross-sea (river) bridges, the great increase in the numbers and the tonnage of ships, and also the extremely weather cause the situation of navigation security increasingly complex, and especially the serious risk events and pollution have the great potential to occur. So strengthening the study of the security of navigation can improve the navigation order, reduce the probability of ship accidents, regulate the behavior of navigation and provide the theoretical support to the further improvement of channel that is crucial to ensure navigational safety and the prevention of water pollution.

At present, the methods of risk assessment for navigation are mainly focused on three aspects: Firstly, determine the security according to the risk assessment criteria of navigation risk factors; secondly, analyze whether a particular channel is prone to have accidents through the analysis of maritime statistical data; thirdly, 
judge whether a particular channel is difficult to navigate using the experience of the driving personnel and the pilot. But, in fact, the security of navigation is a multiple system which is decided by many uncertain factors, such as the flow of ship, the scale of waterway and hydrological conditions, so using only theoretical analysis to solve the problem can't achieve satisfactory results. Furthermore, the lack of statistical data could result in the imperfection in the construction the model and the lack of accurate estimate of artificial judgment, would not really reflect the influence factors and would increases the difficulties in risk evaluation. The navigation security is a vague system, and how to carry out the effective evaluation of the risk of navigation is still in exploration.

Navigation security is decided by many factors such as the channel geometry and its depth, the density of traffic, the aided navigational equipments, the channel environment, the weather condition and the hydrology, the ships' types and scale, the types of accident and the traffic management which have the characteristics of uncertainty, randomness and fuzzy. So the system of navigation security is a typical non-linear system.

\section{Model Selection and Construction}

As to non-linear Dynamics process, whether the traditional safety evaluation methods, Grey system evaluation model, Fuzzy comprehensive assessment model, or Analytic hierarchy process, are unable to solve the following problems: Firstly, these methods can't carry out the real-time analysis; Secondly, the pre-determined factors and the results are mapped to be linear, so it can't reflect the characteristics of study system; Thirdly, once the weights of evaluation factors are determined, they would not vary, but the system is in constant change and development process; Fourthly, they are difficult to get rid of the uncertainty of subjective understanding and ambiguity of participators in the process of safety evaluation.

Artificial Neural Network (ANN) model can overcome the insufficiencies to a certain extent, but it has the disadvantage of slow convergence rate, and easy to fall into a very small value. According to the related simulation results, the series are decomposed by Wavelet having ability to translate and stretch, and can get more approximation of non-linear functions. Therefore, the combination of Wavelet and Neural Network could do better in the ability to approximation, analysis of non-stationary signals, fault tolerance, and construction of nonlinear function model than normal Neural Network.

This article attempts to build the Wavelet Neural Network (WNN) model by Morlet Wavelets Base. The following processes are crucial to the construction of Wavelet Neural Network model.

\section{A. Construction of the transfer function of Wavelet neural network}

If a square integral function satisfies the conditions [1]:

$$
\int_{-\infty}^{\infty}|\varphi(\xi)|^{2}\left|\xi^{-1}\right| d \xi<+\infty
$$

The function $\varphi(t) \in L^{2}(R)$ is Mother-Wavelet. Continuous wavelet $\varphi_{a b}(t)$ can be known as Wavelets Base:

$$
\varphi_{a b}(t)=(\sqrt{|a|})^{-1} \varphi|(t-b) / a|
$$

In (2), $a$ and $b$ can determine the structure of $\varphi_{a b}(t)$. And its wavelet transform can also be expressed as:

$$
k_{f}(a, b)=(\sqrt{|a|})^{-1} \int_{-\infty}^{\infty} f(t) \varphi|(t-b) / a| d t
$$

In (3), $b$ plays a role in translation and $a$ determines the spectrum structure, window size and shape of $\varphi_{a b}(t)$.

Take various $a_{i}$ and $b_{i}$ to determine Wavelets Base $\varphi_{i}(t)$, and use $\varphi_{i}(t)$ as the transfer function: 


$$
\varphi_{i}(t)=\left(\sqrt{\left|a_{i}\right|}\right)^{-1} \varphi\left|\left(t-b_{i}\right) / a_{i}\right|
$$

$\varphi_{i}(t)$ can replace transfer functions such as Sigmoid, and can achieve the optimal approximate of arbitrary function.

B. Model of wavelet neural network safety evaluation

WNN and ANN is similar in structure, and the difference is activation function in the hidden layer. Build WNN (Fig. 1) model having $M$ input nodes and only one output node.

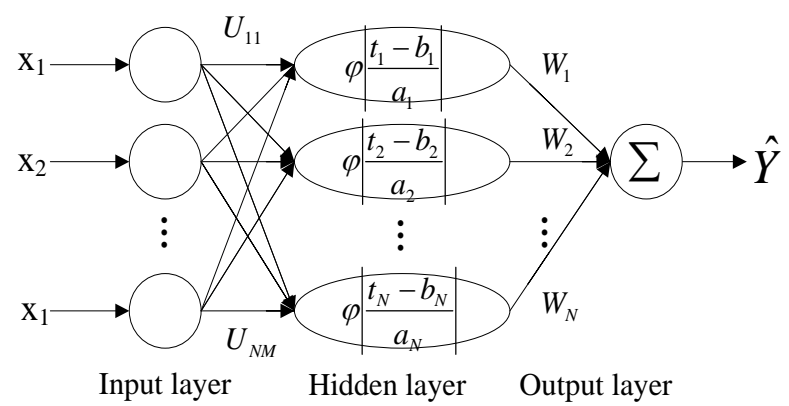

Figure 1. Schematic of wavelet neural network

Thus, the predicted value $\hat{Y}$ can be expressed as the superposition by the limited items of Wavelet Bases [2]:

$$
\hat{Y}=\sum_{i=1}^{N} W_{i} \varphi\left|\frac{t_{i}-b_{i}}{a_{i}}\right|
$$

In (5), $W_{i}$ is the weight of the output of the $i$ Unit.

The input $t_{i}$ of the $i$ Wavelet Bases can be expressed as:

$$
t_{i}=\sum_{j=1}^{M} U_{i j} x_{j}
$$

In (6), $U_{i j}$ is the weight between the input $x_{j}$ of the $j$ unit and the $i$ Wavelet Bases Unit.

So (5) could be transformed as:

$$
\hat{Y}=\sum_{i=1}^{N} W_{i} \varphi\left|\left(\sum_{j=1}^{M} U_{i j} x_{j}-b_{i}\right) / a_{i}\right|
$$

If there are $T$ training samples, $\left(x_{k 1}, x_{k 2}, \cdots, x_{k M}, Y_{k}\right)$

$(k=1,2, \cdots, T)$, we can determine $U_{i j}, a_{i}, b_{i}, W_{i}$ and $N$ through network learning and training.

Use minimum mean square error function to optimize: 


$$
E_{T}=\frac{1}{T} \sum_{k=1}^{T}\left[Y_{k}-\hat{Y}_{k}\right]^{2}
$$

In (8), $N$ could be determined by the testing method. Given the fitting allowed error $\varepsilon$, calculate $E_{i}$ form $N=1$. If $E_{i}<\varepsilon$, takes $N=i$; otherwise, continue to calculate until $N=N^{*}$ and make $E_{N}^{*}<\varepsilon$, so take the value that $N=N^{*}$.

\section{Algorithm of wavelet neural network safety evaluation}

Usually, selects Morlet as the Mother Wavelet [3]:

$$
\varphi(x)=\cos (1.75 x) \cdot e^{-\frac{x^{2}}{2}}
$$

For each given $N,(8)$ can calculate $U_{i j}, a_{i}, b_{i}$ and $W_{i}$. Use Back-Propagation Neural Network training algorithm to adjust the parameters and the weights of the wavelet network. Specific learning algorithm is as following [4]:

1) Build the model of WNN according to security assessment index system of the study objects

2) Initialize the parameters of WNN

Give random initial values of scale factor $a_{i}$, translation factor $b_{i}$, the weight $U_{i j}$ of network nodes and $W_{i}$.

3) Deal with the learning samples

Input the learning samples $x_{k j}(j=1,2, \cdots M ; k=1, \cdots$

$T$ ) and the corresponding expected output $Y_{k}$.

4) Process of self-learning of Wavelet Neural Network

The output of WNN can be calculated using (7).

5) Use the steepest descent method to modify

$$
\begin{aligned}
& \hat{U}_{i j}=U_{i j}-\eta \frac{\partial E_{Q}}{\partial U_{i j}}+\alpha \Delta U_{i j} ; \\
& \hat{W}_{i}=W_{i}-\eta \frac{\partial E_{Q}}{\partial W_{i}}+\alpha \Delta W_{i} ; \\
& \hat{a}_{i}=a_{i}-\eta \frac{\partial E_{Q}}{\partial a_{i}}+\alpha \Delta a_{i} ; \\
& \hat{b}_{i}=b_{i}-\eta \frac{\partial E_{Q}}{\partial b_{i}}+\alpha \Delta b_{i} .
\end{aligned}
$$

In (10), (11), (12) and (13), $\eta$ is learning rate and $\alpha$ is momentum factor.

6) Circulation and termination

When errors are less than the pre-set values or learning step numbers reach the maximum, then stop. Otherwise return to (2). 


\section{Construction the evaluation model for navigation using WNN}

\section{Establishment index system of navigation risk evaluation}

The index system includes the parameters of characteristic and status. The parameters of characteristic could reflect the security of the study object. The parameters of status include the output nodes, the actual meaning and expression of nodes.

\section{E. Build the structure of the wavelet neural network}

Determine the topology structure includes the hidden layer, the nodes of the input and output layers. The number of neurons in the input and output layers is defined according to the evaluation object, and the number in hidden layers can be determined according to the learning and training results [5].

\section{F. $\quad$ Selection and dealing with the learning samples}

Select multiple sets of the status parameters as the learning samples for learning after pretreatment. These samples should reflect the various safety status. The process of WNN learning is determining the connection weights and modifying errors.

\section{G. Establishment the knowledge base}

Through the network learning, confirm input, output, the hidden nodes and network weights of WNN, and build the knowledge base with reasoning mechanisms.

\section{H. Carrying out the safety evaluation of navigation.}

Trained WNN can convert the characteristic values. Enter these values into WNN, and we could get the actual results of system security by knowledge base. The results used as new learning samples can further enrich the knowledge base.

\section{Practical application}

The navigation of Nanjing Yangtze River is selected to carry out the risk evaluation, because it is in a very complicated situation such as various ship types and tonnage, the fluctuation of traffic, geographic environment. The paper builds the index system having 20 items form $X_{1}$ to $X_{20}$ shown in table I [6].

TABLE I. THE PARAMETERS OF WAVELET NEURAL NETWORK INPUT

\begin{tabular}{|c|c|c|c|}
\hline $\mathrm{Xi}$ & Explanation & $\mathrm{Xi}$ & Explanation \\
\hline$X_{I}$ & $\begin{array}{c}\text { the days of visible distance } \\
\text { below } 1,000 \text { meters }\end{array}$ & $X_{11}$ & the height of wave \\
\hline$X_{2}$ & the days of standard wind & $X_{12}$ & the distance of obstacles \\
\hline$X_{3}$ & maximum of flow velocity & $X_{13}$ & the number of obstacles \\
\hline$X_{4}$ & average of flow velocity & $X_{14}$ & the flow of ship traffic \\
\hline$X_{5}$ & maximum of wind velocity & $X_{15}$ & $\begin{array}{l}\text { the convergence points of } \\
\text { ship traffic }\end{array}$ \\
\hline$X_{6}$ & $\begin{array}{l}\text { maximum angle between } \\
\text { the maximum flow and } \\
\text { the main channel }\end{array}$ & $X_{16}$ & $\begin{array}{l}\text { the number of steering } \\
\text { points of ship traffic }\end{array}$ \\
\hline$X_{7}$ & the length of channel & $X_{17}$ & the settings of navigation \\
\hline$X_{8}$ & $\begin{array}{l}\text { the maximum bending of } \\
\text { Channel }\end{array}$ & $X_{18}$ & the condition of pilot \\
\hline
\end{tabular}




\begin{tabular}{|c|c|c|c|}
\hline$X_{9}$ & $\begin{array}{c}\text { the number of } \\
\text { cross-channel points }\end{array}$ & $X_{19}$ & $\begin{array}{c}\text { the supervision of } \\
\text { maritime safety } \\
\text { administration }\end{array}$ \\
\hline$X_{10}$ & the saturation of channel & $X_{20}$ & emergency and rescue \\
\hline
\end{tabular}

According to the relevant investigate and research, we divide the level of navigation security into 'excellent, good, ordinary, poor'. The defining the division criterion of navigation security is shown in table II [7].

TABLE II. THE DIVISION CRITERION OF NAVIGATION SECURITY

\begin{tabular}{|l|c|c|c|c|}
\hline Security & excellent & good & ordinary & poor \\
\hline Scores & $0.9 \sim 1$ & $0.75 \sim 0.9$ & $0.6 \sim 0.75$ & $0 \sim 0.6$ \\
\hline
\end{tabular}

The following step is defining the criterion of the input parameters of assessment system shown in table III.

TABLE III. THE CRITERION OF THE INPUT PARAMETERS OF ASSESSMENT SYSTEM

\begin{tabular}{|c|c|c|c|c|}
\hline Grade & excellent & good & ordinary & poor \\
\hline$X_{I}$ (days) & $<25$ & $25 \sim 45$ & $45 \sim 60$ & $>60$ \\
\hline$X_{2}$ (days) & $<35$ & $35 \sim 60$ & $60 \sim 100$ & $>100$ \\
\hline$X_{3}$ (knot) & $<0.5$ & $0.5 \sim 2.5$ & $2.5 \sim 6.5$ & $>6.5$ \\
\hline$X_{4}$ (knot) & $<0.2$ & $0.2 \sim 0.8$ & $0.8 \sim 1.8$ & $>1.8$ \\
\hline$X_{5}$ (m/.s) & $<10$ & $10 \sim 25$ & $25 \sim 35$ & $>35$ \\
\hline$X_{6}$ (degree) & $0 \sim 10$ & $10 \sim 30$ & $30 \sim 60$ & $>60$ \\
\hline$X_{7}$ (km) & $<30$ & $30 \sim 60$ & $60 \sim 100$ & $>100$ \\
\hline$X_{8}$ (degree) & $0 \sim 15$ & $15 \sim 30$ & $30 \sim 55$ & $>55$ \\
\hline$X_{9}$ (number) & $<1$ & $1 \sim 3$ & $3 \sim 8$ & $>8$ \\
\hline$X_{10}(\%)$ & $<0.7$ & $0.7 \sim 0.8$ & $0.8 \sim 0.9$ & $>0.9$ \\
\hline$X_{11}$ (meter) & $<2.5$ & $2.5 \sim 5.0$ & $5.0 \sim 7.0$ & $>7.0$ \\
\hline$X_{12}$ (meter) & $>300$ & $300 \sim 200$ & $200 \sim 50$ & $<50$ \\
\hline$X_{13}$ (number) & $<1$ & $1 \sim 3$ & $3 \sim 9$ & $>9$ \\
\hline$X_{14}$ (n/d) & $<100$ & $100 \sim 300$ & $300 \sim 900$ & $>900$ \\
\hline$X_{15}$ (number) & $<1$ & $1 \sim 3$ & $3 \sim 8$ & $>8$ \\
\hline$X_{16}$ (number) & $<1$ & $1 \sim 4$ & $4 \sim 9$ & $>9$ \\
\hline
\end{tabular}

The channel of Nanjing Yangtze river is divided into ten parts. Select expert evaluation as samples (table $\mathrm{IV})$ in which the $1 \sim 8$ samples are used as the learning and the $9 \sim 10$ as testing.

TABLE IV. THE SAMPLES OF THE WAVELET NEURAL NETWORK

\begin{tabular}{|c|c|c|c|c|c|c|c|c|c|c|}
\hline $\mathrm{Xi}$ & 1 & 2 & 3 & 4 & 5 & 6 & 7 & 8 & 9 & 10 \\
\hline $\mathrm{X}_{1}$ & 0.87 & 0.75 & 0.86 & 0.81 & 0.76 & 0.69 & 0.79 & 0.80 & 0.87 & 0.76 \\
\hline $\mathrm{X}_{2}$ & 0.82 & 0.93 & 0.86 & 0.72 & 0.86 & 0.81 & 0.75 & 0.72 & 0.91 & 0.85 \\
\hline $\mathrm{X}_{3}$ & 0.78 & 0.76 & 0.86 & 0.82 & 0.92 & 0.88 & 0.84 & 0.72 & 0.68 & 0.90 \\
\hline $\mathrm{X}_{4}$ & 0.72 & 0.77 & 0.75 & 0.85 & 0.89 & 0.69 & 0.55 & 0.89 & 0.81 & 0.92 \\
\hline $\mathrm{X}_{5}$ & 0.85 & 0.76 & 0.86 & 0.69 & 0.68 & 0.83 & 0.73 & 0.77 & 0.87 & 0.82 \\
\hline $\mathrm{X}_{6}$ & 0.75 & 0.84 & 0.79 & 0.88 & 0.90 & 0.69 & 0.71 & 0.73 & 0.84 & 0.82 \\
\hline $\mathrm{X}_{7}$ & 0.88 & 0.82 & 0.79 & 0.75 & 0.90 & 0.81 & 0.79 & 0.83 & 0.76 & 0.76 \\
\hline $\mathrm{X}_{8}$ & 0.68 & 0.77 & 0.69 & 0.68 & 0.75 & 0.72 & 0.82 & 0.80 & 0.76 & 0.85 \\
\hline $\mathrm{X}_{9}$ & 0.72 & 0.76 & 0.84 & 0.82 & 0.79 & 0.82 & 0.76 & 0.78 & 0.92 & 0.73 \\
\hline $\mathrm{X}_{10}$ & 0.86 & 0.87 & 0.81 & 0.89 & 0.82 & 0.78 & 0.92 & 0.69 & 0.75 & 0.87 \\
\hline $\mathrm{X}_{11}$ & 0.76 & 0.84 & 0.81 & 0.79 & 0.88 & 0.91 & 0.75 & 0.76 & 0.71 & 0.68 \\
\hline $\mathrm{X}_{12}$ & 0.69 & 0.74 & 0.79 & 0.75 & 0.81 & 0.86 & 0.82 & 0.87 & 0.69 & 0.90 \\
\hline
\end{tabular}




\begin{tabular}{|c|c|c|c|c|c|c|c|c|c|c|}
\hline $\mathrm{X}_{13}$ & 0.75 & 0.81 & 0.88 & 0.80 & 0.72 & 0.68 & 0.89 & 0.69 & 0.68 & 0.74 \\
\hline $\mathrm{X}_{14}$ & 0.68 & 0.74 & 0.79 & 0.81 & 0.88 & 0.82 & 0.75 & 0.78 & 0.69 & 0.76 \\
\hline $\mathrm{X}_{15}$ & 0.79 & 0.75 & 0.86 & 0.79 & 0.90 & 0.72 & 0.71 & 0.69 & 0.84 & 0.85 \\
\hline $\mathrm{X}_{16}$ & 0.66 & 0.59 & 0.67 & 0.70 & 0.72 & 0.76 & 0.80 & 0.80 & 0.76 & 0.69 \\
\hline $\mathrm{X}_{17}$ & 0.69 & 0.70 & 0.72 & 0.75 & 0.80 & 0.90 & 0.85 & 0.76 & 0.74 & 0.80 \\
\hline $\mathrm{X}_{18}$ & 0.70 & 0.72 & 0.71 & 0.86 & 0.89 & 0.81 & 0.79 & 0.69 & 0.90 & 0.82 \\
\hline $\mathrm{X}_{19}$ & 0.69 & 0.59 & 0.75 & 0.80 & 0.72 & 0.68 & 0.76 & 0.81 & 0.86 & 0.88 \\
\hline $\mathrm{X}_{20}$ & 0.71 & 0.76 & 0.78 & 0.81 & 0.86 & 0.87 & 0.89 & 0.81 & 0.85 & 0.67 \\
\hline Scores & 0.82 & 0.89 & 0.72 & 0.76 & 0.69 & 0.78 & 0.85 & 0.77 & 0.82 & 0.76 \\
\hline
\end{tabular}

According to the index system and the actual investigation, determine WNN topology for $(20,3,1)$, that is with 3 layers, 20 neurons of the input layer, 1 neuron of the output layer. Meanwhile, settle $\eta=0.4, \alpha=0.2$, $\varepsilon=0.0001$.

Apply MATLAB7.0 to train WNN and simulate the process [8]. This article applies WNN and the traditional ANN with the same learning rate, learning times and the output error to learn and train, and then compare the two models.

The error curves are shown as following. From the figure, we can see the convergence rate of WNN is fast than ANN.

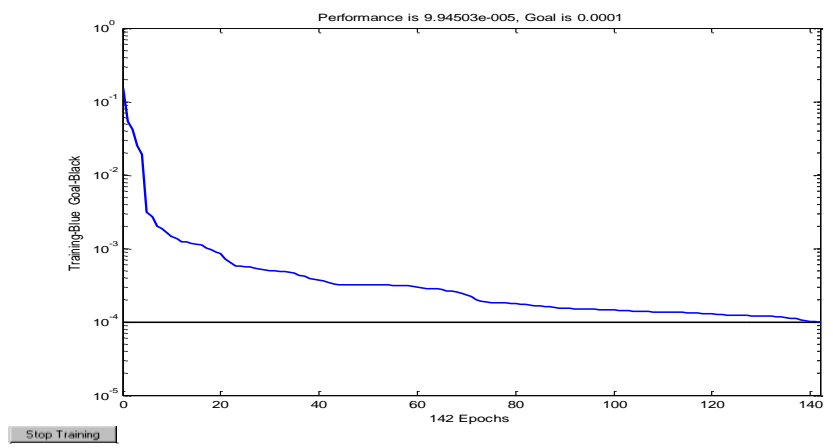

Figure 2. The error curve of wavelet neural network

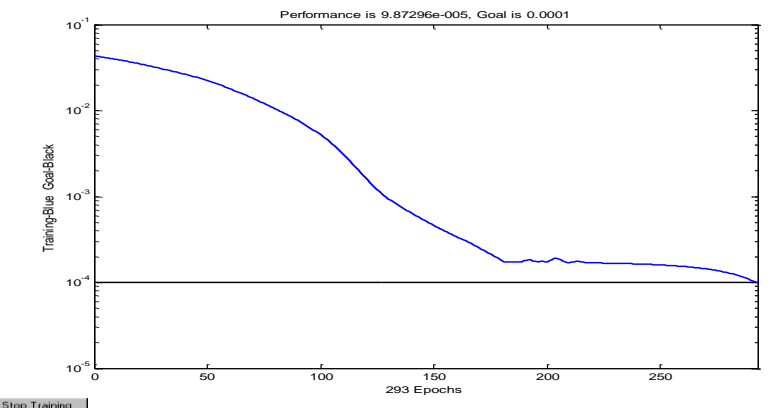

Figure 3. The error curve of artificial neural network

The results show that the relative errors of WNN is less than ANN shown in table $\mathrm{V}$. Therefore, WNN is superior to ANN in higher precision and the ability of approximation. 
TABLE V. COMPARISON THE RESULTS USING WNN AND ANN

\begin{tabular}{|c|c|c|c|c|c|}
\hline Samples & $\begin{array}{c}\text { Date of } \\
\text { samples }\end{array}$ & $\begin{array}{c}\text { Results of } \\
\text { WNN }\end{array}$ & $\begin{array}{c}\text { Results of } \\
\text { ANN }\end{array}$ & $\begin{array}{c}\text { Error of } \\
\text { WNN }\end{array}$ & $\begin{array}{c}\text { Error of } \\
\text { ANN }\end{array}$ \\
\hline 9 & 0.82 & 0.8163 & 0.8286 & $0.45 \%$ & $1.04 \%$ \\
\hline 10 & 0.76 & 0.7613 & 0.7712 & $0.17 \%$ & $1.47 \%$ \\
\hline
\end{tabular}

\section{Conclusion}

This paper puts forward a model for navigation safety assessment based on wavelet neural network, use the parameters affecting the navigation security and the corresponding evaluation results as the training sample, and map the relationship between the state parameter and the status of the safety. The results can well reflect the actual security situation, and show that the method is feasible.

Using wavelet neural network can not only make the comprehensive evaluation of the security of the system, but also can avoid the defects of traditional safety assessment methods in the process of development. Therefore it can realize the dynamic evaluation of the system. At the same time, the introduction of the expansion factor and the translation factor made WNN better than ANN in the ability of function approximation, tolerance and the rate of convergence.

Although this article is focused on the navigation of Nanjing Yangtze River, the model and the algorithm are universal, and can apply to the navigation safety evaluation in other areas. How to comprehensively consider the working environment, human factor and management is the next step to continue.

\section{References}

[1] Ehsan Hossaini-asl, Mehdi Shahbazian, "Nonlinear dynamic system control using wavelet neural network based on sampling theory," Proceedings of the 2009 IEEE International Conference on Systems, Man, and Cybernetics, vol.5, 2009,pp.4502-4507.

[2] Xu Yangwen, "Study on Fault Diagnosis of Rotating Machinery Based on Wavelet Neural Network," 2009 International Conference on Information Technology and Computer Science, vol.3, 2009, pp.221-224.

[3] M. Norgaard, O.H. Ravn, N.K. Poulsen, L.K. Hansen, "Neural Networks for modeling and control of Dynamic Systems"(Springer, 2003).

[4] OUSSAR Y, RIVALS I, PERSONNAZ L, "Training wavelet neural networks for non-linear dynamic input-output modeling," Neuro computing, Vol. 20, Issue 1-3, 1998, pp. 173-188.

[5] Han Liqun, Artificial Neural Network Theory And Application, Design and Applications. Beijing:Chemical Industry Press, 2002.(in Chinese)

[6] ZHAO Zong-fang, "On beacon navigation and hydrographic mapping site management and information dissemination," China State Navigation Institute beacon professional Committee Surveying Group Symposium on academicexchanges collection, Vol. 3,2006,pp.243-246. (in Chinese)

[7] QIU Yun-ming, CHEN Wei-jiong, CHEN Jing-biao, "Comprehensive Environment Assessment of Navigation Safety in River Port Channels by Sea," Navigation of china, Vol. 12, 2005, pp.41-45. (in Chinese)

[8] Cong Shuang, The Theory and Application of Artificial Neural Network using MATLAB, Hefei: China Science \& Technology University Press, 2009. (in Chinese) 